\title{
The "inversion relation" method for obtaining the free energy of the chiral Potts model
}

\author{
R.J. Baxter* \\ Mathematical Sciences Institute and Theoretical Physics, I.A.S. \\ Building 27, The Australian National University, Canberra, A.C.T. 0200, Australia ${ }^{\dagger}$
}

November 29, 2018

\begin{abstract}
We derive the free energy of the chiral Potts model by the infinite lattice "inversion relation" method. This method is non-rigorous in that it always needs appropriate analyticity assumptions. Guided by previous calculations based on exact finite-lattice functional relations, we find that in addition to the usual assumption that the free energy be analytic and bounded in some principal domain of the rapidity parameter space that includes the physical regime, we also need a much less obvious symmetry. We can then obtain the free energy by Wiener-Hopf factorization in the complex planes of appropriate variables. Together with the inversion relation, this symmetry relates the values of the free energy in all neighbouring domains to those in the principal domain.
\end{abstract}

PACS: 05.50.+q, 02.30.Gp

Keywords: Statistical mechanics, lattice models, chiral Potts model, free energy

\section{Introduction}

Onsager calculated the free energy of the two-dimensional Ising model by setting up an algebra that contained the row-to-row transfer matrix [1]. Kaufman simplified the derivation by using spinor operators (i.e. a Clifford algebra)[2], and Kasteleyn showed that the result could be obtained quite directly by pfaffians [3].

Most other solvable models do not appear to be amenable to such algebraic (in particular free-fermionic) methods. For these there are

*Fax: 6126125 5549; e-mail: rj.baxter@apex.net.au.

†This work supported in part by the Australian Research Council. 
three main methods of approach (excluding the Bethe ansatz eigenvector method): the first and third depend explicitly on the Yang-Baxter or star-triangle relation, the second implicitly.

The first is to set up functional relations for the transfer matrix $T$. These define all the eigenvalues of $T$ (for a finite system), so in principle give the exact free energy for a lattice of $M$ rows and $L$ columns. Usually one can solve these explicitly only in the large-lattice limit.

The second is the inversion relation method, due to Stroganov [4]. In this one writes down rotation and inversion relations satisfied by the free energy of the infinite system. They are functional relations (sometimes called difference equations), wherein the free energy is regarded as a function of the rapidity variables (or of their difference, which is the spectral variable). These relations do not by themselves define the free energy, any more than the relation $f(z+1)=2-$ $f(z)$ defines a function $f(z)$ in the complex plane. It is essential to make appropriate analyticity assumptions to complete the relations: for instance in our simple example, if we know that $f(z)$ is analytic and bounded in the vertical strip $0 \leq \Re(z) \leq 1$, then it must be analytic and bounded in the whole plane. By Liouville's theorem it is therefore a constant, so $f(z)=1$.

The third method depends on using the star-triangle relation to relate the free energies of the triangular and honeycomb lattices, differentiating this and using the fact that the derivatives are local correlations that depend only on two rapidities, rather than three. This gives a partial differential equation for the derivatives. As far as the author is aware, this method has been applied only to the Ising and chiral Potts models $[5,6]$.

The free energy of the chiral Potts model has been obtained only by the first and third methods. The result of the first $[7,8]$ is an explicit double integral, while the second [6] gives a hierarchy of equations that implicitly define the free energy (and can be used to obtain the critical exponent $\alpha$ ). Only recently [9] has it been verifed that the two results are equivalent.

The object of this paper is to use the second method - the inversion relation method - to obtain the free energy of the $N$-state chiral Potts model in the infinite-lattice limit. We do this by starting from the functional relations of method 1, taking the large-lattice limit, and showing that

a) the resulting equations can be derived from the inversion and rotation relations,

b) they can be solved by making certain analyticity assumptions. In fact we know these assumptions are valid from method 1, but we offer plausibility arguments in their favour that are independent of method 1.

We then use Wiener-Hopf factorization methods to solve these infinite-lattice functional relations, and inevitably obtain the double integral result of method 1 . 
In later papers we intend to further investigate the analyticity properties of the free energy, or rather of the partition-function per site $\kappa$, as a function of the two rapidity variables $p$ and $q$. It is a meromorphic function on a Riemann surface of infinite genus. Each sheet of this surface can be associated with a point on a lattice in $2 N$ - 1-dimensional space (for $N>2$ ). If one fixes one of the two rapidities $p$ or $q$, then the remaining single-rapidity space is only $N$ dimensional. (The fact that the two-rapidity surface is only $2 N-1$ dimensional, rather than $2 N$, is a manifestation of a weak "rapidity difference property". Unlike other planar models, it is not obvious that this property is particularly helpful.)

This surface is not (for $N>2$ ) contained in the surface generated by the hyperelliptic function variables known to be associated with the chiral Potts model [10]. Thus it is not a single valued-function of these particular variables, so cannot be immediately expressed in terms of hyperelliptic functions of them. However, the surface for the associated function $\tau_{2}\left(t_{q}\right)$ is associated with a space of one dimension less, and is the space spanned by the hyperelliptic variables (after a simple transformation), so hyperelliptic functions may still be useful in this problem.

There are two reasons for this investigation. One is that a large amount of fascinating mathematics has grown out of solvable models, much of it connected with the explicit Jacobi elliptic function parametrization that is used in simpler models such as hard hexagons [11]. Does this generalize to the chiral Potts model and its associated hyperelliptic function parametrization?

The other reason is the continuing problem of the intriguing conjecture for the spontaneous magnetizations of the chiral Potts model $[12,13,14]$. This remains unproven, but there is an infinite-lattice functional relation for it $[15,16,17]$ which is much like the inversion relation for the free energy. If one knows how to solve the latter, then one might hope to use similar techniques to solve the former, and hence verify the conjecture. Only time will tell!

One interesting result in this paper is (71): an unexpected simple symmetry relation satisfied by the free energy of the chiral Potts model.

\section{Functional relations for the transfer matrices}

Consider the square lattice $\mathcal{L}$, drawn diagonally as in Figure 1, with $2 M$ rows of $L$ sites and periodic boundary conditions. At each site $i$ there is a "spin" $\sigma_{i}$, which takes the values $0, \ldots, N-1$. Adjacent spins $i$ and $j$ (with $i$ below $j$ ) interact with Boltzmann weight $W_{p q}\left(\sigma_{i}-\right.$ $\left.\sigma_{j}\right)$ for $\mathrm{SW} \rightarrow \mathrm{NE}$ edges, $\bar{W}_{p q}\left(\sigma_{i}-\sigma_{j}\right)$ for $\mathrm{SE} \rightarrow \mathrm{NW}$ edges. The parameters $p, q$ will now be defined. 


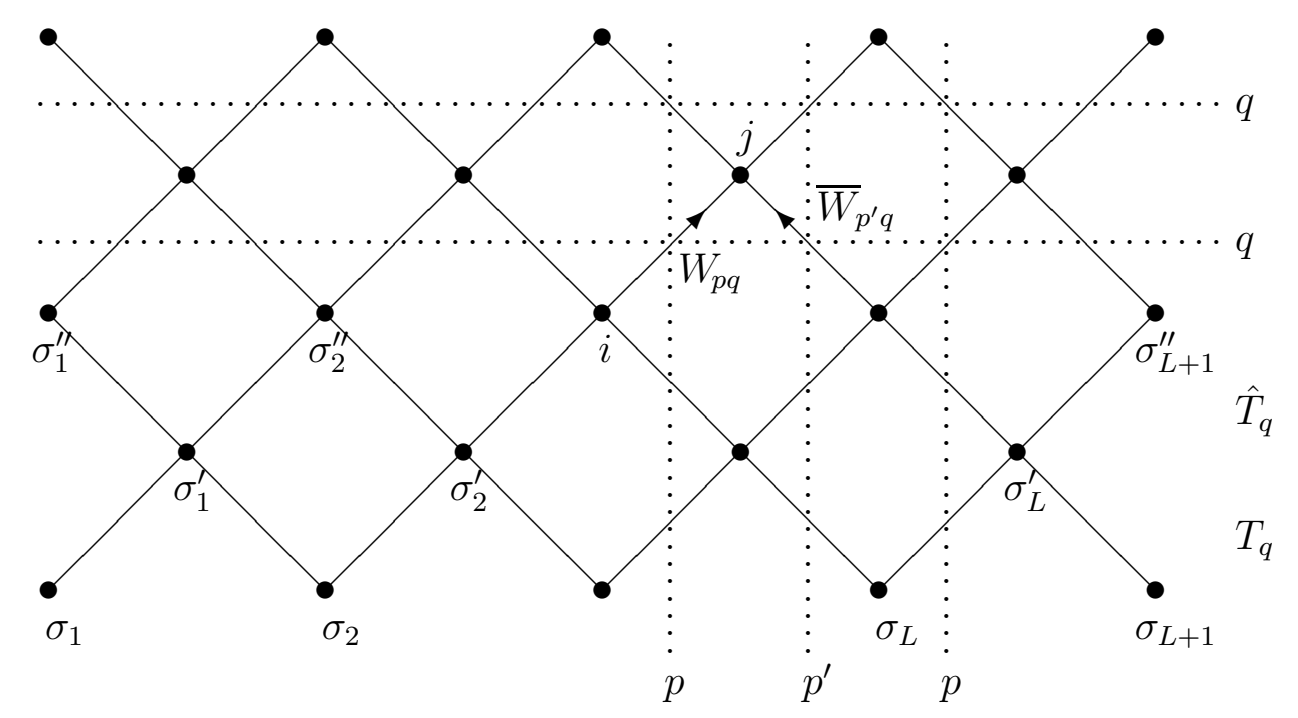

Figure 1: The square lattice $\mathcal{L}$ of $2 M(=4)$ rows with $L$ sites per row. $T_{q}$ is the transfer matrix of an odd row, $\hat{T}_{q}$ of an even row. Three vertical and two horizontal dotted rapidity lines are shown.

Let $\omega=e^{2 \pi i / N}$ be the primitive $N$ th root of unity, and take $k, k^{\prime}$ to be two positive real constants, with $k, k^{\prime}<1$ such that $k^{2}+{k^{\prime}}^{2}=1$. Also, let $p=\left\{x_{p}, y_{p}, \lambda_{p}, t_{p}, \mu_{p}\right\}$ be a set of complex parameters (" $p$ variables") related by

$$
\begin{gathered}
k x_{p}^{N}=1-k^{\prime} / \lambda_{p}, \quad k y_{p}^{N}=1-k^{\prime} \lambda_{p}, \\
x_{p}^{N}+y_{p}^{N}=k\left(1+x_{p}^{N} y_{p}^{N}\right), \quad t_{p}=x_{p} y_{p} \quad, \quad \lambda_{p}=\mu_{p}^{N} .
\end{gathered}
$$

Only one of these variables is independent. In terms of the $a_{p}, b_{p}, c_{p}, d_{p}$ of ref. [18], $x_{p}=a_{p} / d_{p}, y_{p}=b_{p} / c_{p}, \mu_{p}=d_{p} / c_{p}$. We can regard $p$ as a point on an algebraic curve in $\left(x_{p}, y_{p}, \lambda_{p}, t_{p}, \mu_{p}\right)$-space, and refer to it as a "rapidity".

Similarly, define " $q$-variables" $q=\left\{x_{q}, y_{q}, \lambda_{q}, t_{q}, \mu_{q}\right\}$. Then the Boltzmann weight functions are

$$
\begin{gathered}
W_{p q}(n)=W_{p q}(n+N)=\left(\mu_{p} / \mu_{q}\right)^{n} \prod_{j=1}^{n}\left(y_{q}-\omega^{j} x_{p}\right) /\left(y_{p}-\omega^{j} x_{q}\right) \\
\left.\bar{W}_{p q}(n)=\bar{W}_{p q}(n+N)=\left(\mu_{p} \mu_{q}\right)^{n} \prod_{j=1}^{n}\left(\omega x_{p}-\omega^{j} x_{q}\right) /\left(y_{q}-\omega^{j} y_{p}\right) 3 .\right)
\end{gathered}
$$

Note that $W_{p q}(0)=\bar{W}_{p q}(0)=1$. We use this normalization throughout this paper. If $x_{p}, x_{q}, y_{p}, y_{q}, \omega x_{p}$ all lie on the unit circle and are ordered anti-cyclically round it, then the Boltzmann weights 
$W_{p q}(n), \bar{W}_{p q}(n)$ are real and positive for all integers $n$. We refer to this case as the physical regime.

If $Z$ is the usual partition function, in the physical regime we expect the limit

$$
\kappa_{p q}=\lim _{\mathcal{N} \rightarrow \infty} Z^{1 / \mathcal{N}}
$$

to exist, where $\mathcal{N}$ is the number of sites of the lattice, and the limit is to be taken so that the lattice is infinitely large in all directions. We refer to $\kappa_{p q}$ as the partition function per site. The free energy per site is

$$
F_{p q}=-k_{B} \mathcal{T} \log \kappa_{p q}
$$

$k_{B}$ being Boltzmann's constant and $\mathcal{T}$ the temperature. Having taken this limit in the physical regime, we then define $\kappa_{p q}$ outside the regime by analytic continuation. We shall find that the resulting function $\kappa_{p q}$ lives on an infinite Reimann surface.

Each edge of the lattice can be regarded as associated with two rapidity lines, one vertical and one horizontal, as in Figure 1. The rapidities may be different for different lines. In $3, p$ is the rapidity of the vertical line through the edge being considered, $q$ is the rapidity of the horizontal line.

As in [19], we distinguish alternate columns of the lattice, assigning them vertical rapidities $p$ and $p^{\prime}$ as in Figure 1. Then, as in [19], we can define two $N^{L}$ by $N^{L}$ row-to-row transfer matrices $T, \hat{T}$, corresponding to the two types of row of the lattice. They have elements

$$
\begin{aligned}
& T_{\sigma, \sigma^{\prime}}=\prod_{j=1}^{L} W_{p q}\left(\sigma_{j}-\sigma_{j}{ }^{\prime}\right) \bar{W}_{p^{\prime} q}\left(\sigma_{j+1}-\sigma_{j}{ }^{\prime}\right), \\
& \hat{T}_{\sigma, \sigma^{\prime}}=\prod_{j=1}^{L} \bar{W}_{p q}\left(\sigma_{j}-\sigma_{j}{ }^{\prime}\right) W_{p^{\prime} q}\left(\sigma_{j}-\sigma_{j+1}{ }^{\prime}\right) .
\end{aligned}
$$

If there are $M$ rows of each type, then the partition function is

$$
Z=\operatorname{Trace}(T \hat{T})^{M} .
$$

We regard $p$ and $p^{\prime}$ as given, and view $T, \hat{T}$ as functions of the horizontal rapidity $q$. The parameter $\mu_{q}$ enters the rhs of (6) only via its $N$ th power, so they are uniqely defined if both $x_{q}$ and $y_{q}$ are prescribed. We write them as $T\left(x_{q}, y_{q}\right), \hat{T}\left(x_{q}, y_{q}\right)$.

Some scalar functions of $q$ that we shall need are

$$
\begin{gathered}
z\left(t_{q}\right)=\left[\omega \mu_{p} \mu_{p^{\prime}}\left(t_{p}-t_{q}\right)\left(t_{p^{\prime}}-t_{q}\right) /\left(y_{p} y_{p^{\prime}}\right)^{2}\right]^{L} \\
z_{j}\left(t_{q}\right)=z\left(t_{q}\right) z\left(\omega t_{q}\right) \cdots z\left(\omega^{j-1} t_{q}\right) \\
\alpha_{q}=\alpha\left(\lambda_{q}\right)=\left[\frac{k^{\prime}\left(1-\lambda_{p} \lambda_{q}\right)\left(1-\lambda_{p^{\prime}} \lambda_{q}\right)}{k^{2} \lambda_{q} y_{p}^{N} y_{p^{\prime}}^{N}}\right]^{L}=\left[\frac{\left(y_{p}^{N}-x_{q}^{N}\right)\left(t_{p^{\prime}}^{N}-t_{q}^{N}\right)}{y_{p}^{N} y_{p^{\prime}}^{N}\left(x_{p^{\prime}}^{N}-x_{q}^{N}\right)}\right]^{L} \\
\bar{\alpha}_{q}=\alpha\left(1 / \lambda_{q}\right), \alpha_{q} \bar{\alpha}_{q}=z_{N}\left(t_{q}\right)
\end{gathered}
$$




$$
\begin{gathered}
r_{p^{\prime}, q}=r_{p^{\prime}}\left(x_{q}, y_{q}\right)=\left\{\frac{N\left(x_{p^{\prime}}-x_{q}\right)\left(y_{p^{\prime}}-y_{q}\right)\left(t_{p^{\prime}}^{N}-t_{q}^{N}\right)}{\left(x_{p^{\prime}}^{N}-x_{q}^{N}\right)\left(y_{p^{\prime}}^{N}-y_{q}^{N}\right)\left(t_{p^{\prime}}-t_{q}\right)}\right\}^{L}, \\
h_{j, q}=\prod_{m=1}^{j-1}\left\{\frac{y_{p} y_{p^{\prime}}\left(x_{p^{\prime}}-\omega^{m} x_{q}\right)}{\left(y_{p}-\omega^{m} x_{q}\right)\left(t_{p^{\prime}}-\omega^{m} t_{q}\right)}\right\}^{L} .
\end{gathered}
$$

We shall also need the $N^{L}$ by $N^{L}$ cyclic shift matrix $X$, with elements

$$
X_{\sigma, \sigma^{\prime}}=\prod_{j=1}^{L} \delta_{\sigma_{j}, \sigma_{j}^{\prime}+1} .
$$

\section{The $T \hat{T}$ relations}

Without loss of generality, we can take the integers $k, l$ of [19] to be $0, j$. Then the functional relations (3.46) therein become

$T\left(x_{q}, y_{q}\right) \hat{T}\left(y_{q}, \omega^{j} x_{q}\right)=r_{p^{\prime}, q} h_{j, q}\left[\tau_{j}\left(t_{q}\right)+z_{j}\left(t_{q}\right) X^{j} \tau_{N-j}\left(\omega^{j} t_{q}\right) / \alpha_{q}\right]$,

for $j=0, \ldots, N$.

Here $\tau_{j}\left(t_{q}\right)$ is the transfer matrix of the associated $\tau_{j}$ model defined in eqns. (3.44) - (3.48) of [19] (which is related to the superintegrable chiral Potts model and to the model whose column transfer matrix is the $Q$ matrix of the six-vertex model [20]). This matrix depends on $q$ only via the parameter $t_{q}$ : in fact it is is a polynomial in $t_{q}$ of degree $(j-1) L$.

\section{The $\tau_{2} T$ relation}

Similarly, equation (4.20) of [19] becomes

$$
\begin{gathered}
\tau_{2}\left(t_{q}\right) T\left(\omega x_{q}, y_{q}\right)=\left[\frac{\left(y_{p}-\omega x_{q}\right)\left(t_{p^{\prime}}-t_{q}\right)}{y_{p} y_{p^{\prime}}\left(x_{p^{\prime}}-x_{q}\right)}\right]^{L} T\left(x_{q}, y_{q}\right)+ \\
{\left[\frac{\omega \mu_{p} \mu_{p^{\prime}}\left(x_{p^{\prime}}-\omega x_{q}\right)\left(t_{p}-\omega t_{q}\right)}{y_{p} y_{p^{\prime}}\left(y_{p}-\omega^{2} x_{q}\right)}\right]^{L} X T\left(\omega^{2} x_{q}, y_{q}\right) .}
\end{gathered}
$$

Two other relations can immediately be obtained from (13) and (14) by interchanging $p$ with $p^{\prime}$ and $T$ with $\hat{T}$, while leaving $\tau_{j}\left(t_{q}\right)$ unchanged. As is shown in (4.22) - (4.30) of [19], one can then deduce from them two sets of relations involving only the functions $\tau_{j}\left(t_{q}\right)$, which we now give. 


\section{The $\tau_{j}$ relations}

The relations (4.27) of [19] are

$$
\begin{gathered}
\tau_{j}\left(t_{q}\right) \tau_{2}\left(\omega^{j-1} t_{q}\right)=z\left(\omega^{j-1} t_{q}\right) X \tau_{j-1}\left(t_{q}\right)+\tau_{j+1}\left(t_{q}\right), \\
\tau_{j}\left(\omega t_{q}\right) \tau_{2}\left(t_{q}\right)=z\left(\omega t_{q}\right) X \tau_{j-1}\left(\omega^{2} t_{q}\right)+\tau_{j+1}\left(t_{q}\right),
\end{gathered}
$$

for $j=1, \ldots, N$, where $\tau_{N+1}\left(t_{q}\right)$ is defined by

$$
\tau_{N+1}\left(t_{q}\right)=z\left(t_{q}\right) X \tau_{N-1}\left(\omega t_{q}\right)+\left(\alpha_{q}+\bar{\alpha}_{q}\right) \tau_{1}\left(t_{q}\right)
$$

and

$$
\tau_{0}\left(t_{q}\right)=0, \tau_{1}\left(t_{q}\right)=1,
$$

i.e. $\tau_{1}\left(t_{q}\right)$ is the identity matrix.

\section{Invariances}

Apart from the relations $k^{2}+{k^{\prime}}^{2}=1, x_{p}^{N}+y_{p}^{N}=k\left(1+x_{p}^{N} y_{p}^{N}\right)$, all the above equations are unchanged by multiplying $x_{p}, x_{q}, y_{p}, y_{q}, t_{p}, t_{q}, k$ by $\epsilon, \epsilon, \epsilon, \epsilon, \epsilon^{2}, \epsilon^{2}, \epsilon^{-N}$, while leaving $\lambda_{p}, \lambda_{q}, \mu_{p}, \mu_{q}$ and the functions $T\left(x_{q}, y_{q}\right), \tau_{j}\left(t_{q}\right), S\left(\lambda_{q}\right)$ unchanged. This invariance persists if in section 3 we write $\eta$ and $1 / \eta$ as $\left[\left(1-k^{\prime}\right) / k\right]^{2 / N}$ and $\left[\left(1+k^{\prime}\right) / k\right]^{2 / N}$, respectively.

Also, $z_{j}\left(t_{q}\right), \alpha_{q}, \bar{\alpha}_{q}, h_{j, q}, \tau_{j}\left(t_{q}\right), \xi_{q}, S\left(\lambda_{q}\right)$ explicitly contain the factors $\beta^{-2 j}, \beta^{-N}, \beta^{-N}, \beta^{j-1}, \beta^{1-j}, \beta^{-N(N-1) / 2}, \beta^{-N(N-1) / 2}$, where $\beta=\left(y_{p} y_{p^{\prime}}\right)^{L}$. These factors are "constants" (independent of $q$ ) and cancel out of the functional relations (13) - (17), so we could have redefined the functions so as to remove them. We prefer to leave them in so as to make the previous invariance more explicit.

\section{Comments}

Because of the star-triangle relation, the matrices $T, \hat{T}$ satisfy the commutation relations (2.32)- (2.33) of [19]. From this it follows that there exist invertible matrices $P_{1}, P_{2}$, independent of the horizontal rapidity $q$, such that $P_{1}^{-1} T\left(x_{q}, y_{q}\right) P_{2}, P_{2}^{-1} \hat{T}\left(x_{q}, y_{q}\right) P_{1}, P_{1}^{-1} \tau_{j}\left(t_{q}\right) P_{1}$ are all diagonal matrices, for all $q$. In this sense the functional relations above can all be simultaneously diagonalized. Their diagonal elements are then just scalar functional relations for each eigenvalue.

Hereinafter we shall we shall work in this diagonal representation. Further, we shall only consider the eigenvalue that is the maximum eigenvalue of $T\left(x_{q}, y_{q}\right) \hat{T}\left(x_{q}, y_{q}\right)$ in the physical regime, so from now on $T\left(x_{q}, y_{q}\right), \hat{T}\left(x_{q}, y_{q}\right), \tau_{j}\left(t_{q}\right)$ are to be interpreted as the functions for this particular eigenvalue. From (4) and (7), noting that the the lattice has $\mathcal{N}=2 L M$ sites, the partition function per site is

$$
\kappa_{p q}=\lim _{L \rightarrow \infty}\left[T\left(x_{q}, y_{q}\right) \hat{T}\left(x_{q}, y_{q}\right)\right]^{1 / 2 L} .
$$


We have distinguished the rapidities $p, p^{\prime}$ of the odd and even vertical rapidity lines as it is easy to go from the above equations to those for a model where the rapidities $p(1), \ldots, p(2 L)$ of the $2 L$ vertical rapidity lines are all different: wherever one sees an expression involving $p, p^{\prime}$ raised to the power $L$, simply replace it by a product over the odd and even rapidities, respectively. Thus (11) becomes

$$
h_{j, q}=\prod_{m=1}^{j-1} \prod_{r=1}^{L}\left\{\frac{y_{p_{2 r-1}} y_{p_{2 r}}\left(x_{p_{2 r}}-\omega^{m} x_{q}\right)}{\left(y_{p_{2-1}-1}-\omega^{m} x_{q}\right)\left(t_{p_{2 r}}-\omega^{m} t_{q}\right)}\right\} .
$$

However, from now on we shall only consider the fully homogeneous model, with vertical rapidity $p^{\prime}=p$ for all columns. In this case $T$ and $\hat{T}$ differ only in a cyclic shift of the $L$ spins, and since we are considering only the maximum eigenvalue, the associated eigenvector is unchanged by such a shift. This eigenvector is also unchanged by multiplication by $X$, so hereinafter we take

$$
p^{\prime}=p, \hat{T}\left(x_{q}, y_{q}\right)=T\left(x_{q}, y_{q}\right), X=1 .
$$

\section{Functional relations in the infinite- lattice limit}

We emphasize that the above equations are exact for a lattice with a finite number $L$ of sites per row. In $[7,8,21]$ they were solved in the limit of $L$ large. A key step in this working was to note that in a selected domain on the $\left(x_{q}, y_{q}\right)$ surface, certain terms in each relation became exponentially small (relative to the other terms) as $L$ became large, so could be neglected.

We can identify such terms by examining the low-temperature limit, when $k^{\prime} \rightarrow 0$ and $k \rightarrow 1$. Similarly to [7], take $y_{p}, y_{q} \rightarrow 1$,

$x_{p} \simeq t_{p}, x_{q} \simeq t_{q}, \lambda_{p}=\mathrm{O}\left(k^{\prime}\right), \lambda_{q}=\mathrm{O}\left(k^{\prime}\right)$. Then $z\left(t_{q}\right)=\mathrm{O}\left(k^{2 L / N}\right)$, and from the definitions in [19],

$$
T\left(x_{q}, y_{q}\right) \simeq 1, \tau_{2}\left(t_{q}\right) \simeq\left(1-\omega t_{q}\right)^{L}, \tau_{j}\left(t_{q}\right) \simeq \mathrm{O}(1) .
$$

It follows that all terms in (15) - (17) that contain the function $z\left(t_{q}\right)$ or $\bar{\alpha}_{q}$ will be negligible, giving

$$
\begin{aligned}
& \tau_{j}\left(t_{q}\right)=\tau_{2}\left(t_{q}\right) \tau\left(\omega t_{q}\right) \cdots \tau_{2}\left(\omega^{j-2} t_{q}\right) \\
& \tau_{2}\left(t_{q}\right) \tau_{2}\left(\omega t_{q}\right) \cdots \tau_{2}\left(\omega^{N-1} t_{q}\right)=\alpha_{q}
\end{aligned}
$$

Similarly, the last term in (14) will be neglible, so

$$
\tau_{2}\left(t_{q}\right)=\left[\frac{\left(y_{p}-\omega x_{q}\right)\left(t_{p}-t_{q}\right)}{y_{p}^{2}\left(x_{p}-x_{q}\right)}\right]^{L} \frac{T\left(x_{q}, y_{q}\right)}{T\left(\omega x_{q}, y_{q}\right)} .
$$


Replacing $x_{q}, y_{q}, t_{q}$ in this equation by $\omega^{j} x_{q}, y_{q}, \omega^{j} t_{q}$ and taking the product over $j=0, \ldots, N-1$, we obtain (23).

The last term on the rhs of (13) will also be neglible, provided $\lambda_{q}=\mathrm{o}\left(k^{(N-2 j) / N}\right)$, i.e. for $j=1, \ldots, N$. (The $j=0$ equation is the same as $j=N$, so we lose nothing by ignoring $j=0$.)

Taking $j=1$, we obtain

$$
T\left(x_{q}, y_{q}\right) T\left(y_{q}, \omega x_{q}\right)=r_{p, q}=r_{p}\left(x_{q}, y_{q}\right) .
$$

Keeping $y_{q}$ fixed, replacing $j$ by $j-1$ and $x_{q}$ by $\omega x_{q}$ in (13), then dividing the resulting equation into the original equation (13), we get

$$
\frac{T\left(x_{q}, y_{q}\right)}{T\left(\omega x_{q}, y_{q}\right)}=\left\{\frac{y_{p}^{2}\left(x_{p}-x_{q}\right)}{\left(y_{p}-\omega x_{q}\right)\left(t_{p}-t_{q}\right)}\right\}^{L} \tau_{2}\left(t_{q}\right)
$$

for $j=2, \ldots, N$. But this is the same as (24), so there is only one further equation contained in the set (13), namely (25). ${ }^{1}$

To summarize: (24) can be regarded as defining $\tau_{2}\left(t_{q}\right)$, and (22) as defining $\tau_{j}\left(t_{q}\right)$. The product relation (23) is a direct consequence of these definitions. So, out of all the functional relations we originally wrote down, the only relation left that contains information about the function $T\left(x_{q}, y_{q}\right)$ is $(25)$.

\section{The function $S\left(\lambda_{q}\right)$}

We shall need another function, also introduced in [7], ${ }^{2}$ namely

$$
S\left(\lambda_{q}\right)=\xi_{q}^{L} T\left(x_{q}, y_{q}\right) T\left(\omega x_{q}, y_{q}\right) \cdots T\left(\omega^{N-1} x_{q}, y_{q}\right)
$$

where

$$
\xi_{q}=\prod_{j=1}^{N-1}\left[\frac{\mu_{p}\left(y_{q}-\omega^{-j} y_{p}\right)\left(y_{q}-\omega^{j+1} x_{p}\right)}{y_{p}^{2}}\right]^{j} .
$$

In $[7,8]$ it is shown, for finite $L$, that $S\left(\lambda_{q}\right)$ is a polynomial in $\lambda_{q}$ of degree $(N-1) L$.

\section{Domains}

The parameters $x_{q}, y_{q}, t_{q}, \lambda_{q}$ are multi-valued functions of one another and we have to be careful to identify them when working with the above equations.

From (1) and (2), $t_{q}$ and $\lambda_{q}$ are related by

$$
k^{2} t_{q}^{N}=1-k^{\prime}\left(\lambda_{q}+\lambda_{q}^{-1}\right)+k^{2} .
$$

\footnotetext{
${ }^{1}$ To put this point another way, in the large lattice limit all the $N+1$ identities (13) can be obtained from (24) and (25).

${ }^{2}$ This definition differs from that in [7], but only in an extra factor $N^{N / 2}$ $(-1)^{(N-2)(N-1) / 2}$.
} 


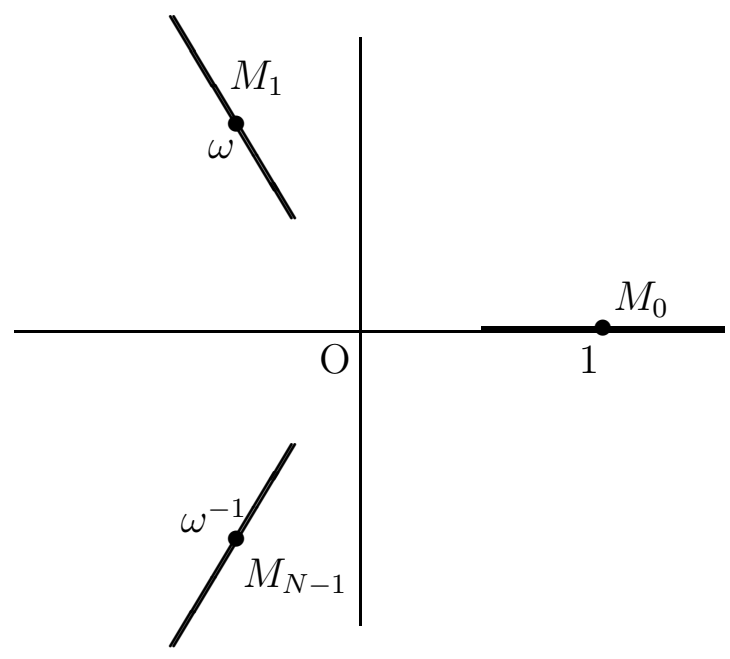

Figure 2: The complex $t_{q}$-plane with its $N$ branch cuts $M_{0}, \ldots, M_{N-1}$

$$
\text { (for } N=3 \text { ). }
$$

Define

$$
\eta=\left[\left(1-k^{\prime}\right) /\left(1+k^{\prime}\right)\right]^{1 / N}, 0<\eta<1,
$$

and consider the cut $t_{q}$-plane shown in Figure 2, with branch points at $t_{q}=\omega^{j} \eta$ and $t_{q}=\omega^{j} / \eta$ and a branch cut $M_{j}$ in between, for $j=0, \ldots N-1$,as shown. Then $\lambda_{q}$ is a single-valued analytic function of $t_{q}$ in this cut plane, and we can require (consistently with the lowtemperature regime considered above) that $\left|\lambda_{q}\right|<1$. We can then choose $y_{q}$ to be the solution of (1) such that $\left|\arg y_{q}\right|<\pi / 2 N$. (If $k^{\prime}$ is small, this means that $y_{q} \simeq 1$.) Finally, we define $x_{q}$ to be $t_{q} / y_{q}$.

The result is that $x_{q}$ lies in the region $\mathcal{E}$ of Figure 3 , while $y_{q}$ lies in the region $\mathcal{R}_{0}$. The boundaries of $\mathcal{R}_{0}, \ldots, \mathcal{R}_{N-1}$ are where $\left|\lambda_{q}\right|=1$.

Let us use the symbol $\mathcal{D}_{0}$ to denote the domain we have just specified, i.e.

$$
\mathcal{D}_{0}: \quad\left|\lambda_{q}\right|<1, x_{q} \in \mathcal{E}, y_{q} \in \mathcal{R}_{0} .
$$

Since $t_{q}$ and $\lambda_{q}$ are uniquely determined if both $x_{q}$ and $y_{q}$ are known, we shall often say that " $\left(x_{q}, y_{q}\right)$ lies in $\mathcal{D}_{0}$ " if the above constraints are satisfied, but it must of course be remembered that $x_{q}, y_{q}$ are related complex variables.

If $k^{\prime}$ is small, $k \simeq 1$ and $\left(x_{q}, y_{q}\right)$ lies in $\mathcal{D}_{0}$, then $y_{q} \simeq 1$ and $x_{q}$ is barred only from small regions about the $N$ points $1, \omega, \ldots \omega^{N-1}$.

If $x_{q}$ is an allowed value in $\mathcal{D}_{0}$, then so are $\omega^{j} x_{q}$, for all integers $j$. We shall sometimes regard $x_{q}$ as the independent variable in $\mathcal{D}_{0}$.

We shall need to consider the domains (Riemann sheets in the $t_{q^{-}}$ variable) neighbouring $\mathcal{D}_{0}$. There are $N$ of these, obtained from $\mathcal{D}_{0}$ by moving $t_{q}$ across one of the $N$ branch cuts in Figure 2. Then $x_{q}$ moves into one of $\mathcal{R}_{0}, \ldots, \mathcal{R}_{N-1}$ in Figure 3 , while $y_{q}$ moves into $\mathcal{E}$. For instance, if we move $t_{q}$ across the cut $M_{r}$ from $\omega^{r} \eta$ to $\omega^{r} / \eta$, we 


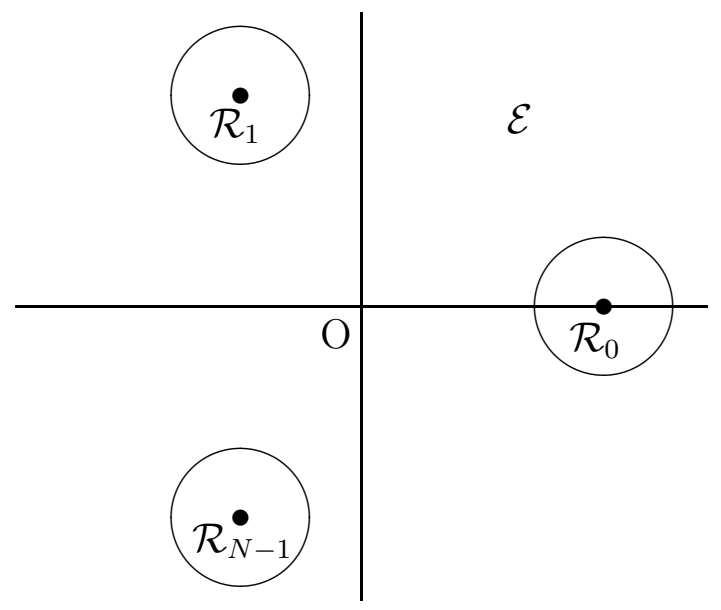

Figure 3: The $N+1$ regions $\mathcal{E}, \mathcal{R}_{0}, \ldots, \mathcal{R}_{N-1}$ of the complex plane in which $x_{q}$ and $y_{q}$ lie (for $N=3$ ). $\mathcal{R}_{0}, \ldots \mathcal{R}_{N-1}$ are the interiors of the approximate circles centred on $1, \omega, \ldots, \omega^{N-1}$. $\mathcal{E}$ is the the complex plane outside all $N$ circles.

enter the domain $\mathcal{D}_{r}^{\prime}$, where

$$
\mathcal{D}_{r}^{\prime}: \quad\left|\lambda_{q}\right|>1, x_{q} \in \mathcal{R}_{r}, y_{q} \in \mathcal{E} .
$$

Now it is $x_{q}$ that is severely restricted,, while $y_{q}$ is free to move about most of the complex plane.

By continuation from the low-temperature, small $k^{\prime}$, limit, the functional relations (22) - (28) hold for $\left(x_{q}, y_{q}\right)$ and $\left(x_{p}, y_{p}\right)$ both lying in $\mathcal{D}_{0}$, except that we must be particularly careful with (25), since the arguments $\left(x_{q}, y_{q}\right),\left(x_{q}^{\prime}, y_{q}^{\prime}\right)=\left(y_{q}, \omega x_{q}\right)$ of the two $T$-functions therein cannot both lie in $\mathcal{D}_{0}$. One of them must lie in a neighbouring domain, but which domain? The answer is that we want both $\left(x_{q}, y_{q}\right)$ and $\left(x_{q}^{\prime}, y_{q}^{\prime}\right)$ to be in or near the physical regime. Only there can we expect to obtain the correct free energy in the large-lattice limit.

If $\left(x_{q}, y_{q}\right)$ is in the physical regime, then, from our remarks before (4), $x_{q}, y_{q}, t_{q}$ lie on the unit circle and

$$
\arg y_{q}-2 \pi / N<\arg x_{q}<\arg y_{q},
$$

If it also in $\mathcal{D}_{0}$, then $y_{q}$ must be inside $\mathcal{R}_{0}$, so $x_{q}$ must be on that portion of the unit circle between between regions $\mathcal{R}_{0}$ and $\mathcal{R}_{N-1}$ in Figure 3. Hence $x_{q}^{\prime} \in \mathcal{R}_{0}$, so $\left(x_{q}^{\prime}, y_{q}^{\prime}\right)$ lies in $\mathcal{D}_{0}^{\prime}$. Writing $T_{r}\left(x_{q}, y_{q}\right)$ for the analytic continuation of $T\left(x_{q}, y_{q}\right)$ to domain $\mathcal{D}_{r}^{\prime}$, we should therefore write (25) as

$$
T\left(x_{q}, y_{q}\right) T_{0}\left(y_{q}, \omega x_{q}\right)=r_{p}\left(x_{q}, y_{q}\right) .
$$

Alternatively, $\left(x_{q}^{\prime}, y_{q}^{\prime}\right)$ may lie in the physical regime and in $\mathcal{D}_{0}$, with $y_{q}^{\prime} \in \mathcal{R}_{0}$. Then $x_{q} \in \mathcal{R}_{N-1}$ and, dropping the suffixes on $\left(x_{q}^{\prime}, y_{q}^{\prime}\right)$, 
(25) becomes

$$
T_{N-1}\left(\omega^{-1} y_{q}, x_{q}\right) T\left(x_{q}, y_{q}\right)=r_{p}\left(\omega^{-1} y_{q}, x_{q}\right) .
$$

Both (33) and (34), with $\left(x_{q}, y_{q}\right) \in \mathcal{D}_{0}$, are valid interpretations of (25).

We refer to $\mathcal{D}_{0}$ as the principal or central domain (or Riemann sheet). In a subsequent paper we intend to discuss the full Riemann surface formed by analytically continuing $\lim _{L \rightarrow \infty} T\left(x_{q}, y_{q}\right)^{1 / L}$ in both the $q$ and the $p$ variables.

\section{The inversion relation}

We now turn from the first to the second general method for calculating free energies, the inversion relation method [4, 22, 23, 24]. Let

$$
\bar{D}_{p q}=\operatorname{det}_{N} \bar{W}_{p q}(i-j), P_{p q}=\prod_{n=0}^{N-1} W_{p q}(n)
$$

i.e. $\bar{D}_{p q}$ is the determinant of the $N$ by $N$ Toeplitz (cyclic) matrix with entry $\bar{W}_{p q}(i-j)$ in row $i$ and column $j$. Then under quite general circumstances the partition function per site $\kappa_{p q}$ satisfies the inversion relation

$$
\kappa_{p q} \kappa_{q p}=\left[\bar{D}_{p q} P_{p q} \bar{D}_{q p} P_{q p}\right]^{1 / N}
$$

For the chiral Potts model $P_{p q} P_{q p}=1$ and (36) becomes, using (2.48) of [19],

$$
\kappa_{p q} \kappa_{q p}=r_{p q}^{1 / L}
$$

where $r_{p q}$ is defined in (10).

Let $R$ be the operator that acts on the rapidity $p$ so that

$$
x_{R p}=y_{p}, y_{R p}=\omega x_{p}, \mu_{R p}=1 / \mu_{p} .
$$

Then replacing $p, q$ by $q, R p$ is equivalent to rotating the lattice through $90^{\circ}$. This does not change the partition function per site, so

$$
\kappa_{p q}=\kappa_{q, R p} .
$$

Combining (37) and (39) gives

$$
\kappa_{p q} \kappa_{p, R q}=r_{p q}^{1 / L}
$$

Remembering that $\kappa_{p q}=T\left(x_{q}, y_{q}\right)^{1 / L}$, it follows that

$$
T\left(x_{q}, y_{q}\right) T\left(y_{q}, \omega x_{q}\right)=r_{p q} .
$$

This is precisely the relation (25), again with one function $T$ being understood to be the analytic continuation of the other from the physical regime. Thus we did not need to go through all the working 
that was necessary in [20], [19] to derive the functional relations (13) (18). We could have used the simple inversion relation, obtained (41), i.e. (25), then defined the auxiliary functions $\tau_{j}\left(t_{q}\right), S\left(\lambda_{q}\right)$ so as to obtain the infinite-lattice relations (22) - (28) above.

\section{Solution of the infinite-lattice func- tional relations}

We now solve the infinite-lattice relations (22) - (28) successively for $\tau_{2}\left(t_{q}\right), S\left(\lambda_{q}\right), T\left(x_{q}, y_{q}\right)$, thereby obtaining the partition function per site $\kappa_{p q}$. Each side of every one of these relations is a quantity raised to the power $L$, and this is the only way $L$ enters the relations. We should define new functions that are the $L$ th roots of $\tau_{2}\left(t_{q}\right), S\left(\lambda_{q}\right)$, $T\left(x_{q}, y_{q}\right)$, and write down the $L$ th roots of each relation. Equivalently, from now on we formally set

$$
L=1
$$

in equations (8) - (11) and (21) - (41).

Thus $T\left(x_{q}, y_{q}\right)$, or $T_{p q}$, is now the partition function per site $\kappa_{p q}$ of the lattice, defined as in the paragraph containing (4), (5), i.e.

$$
\kappa_{p q}=T_{p q}=T\left(x_{q}, y_{q}\right) .
$$

Our derivation will be based solely on the inversion relation (25), as interpreted in (33), and the definitions in (22) - (28). It will necessarily involve various analyticity assumptions, and in making these we have been heavily guided by the previous derivations in [7], [8]. In particular, we shall regard $\tau_{2}\left(t_{q}\right)$ as a function of $t_{q}$ (rather than, say, $x_{q}$ or $\mu_{q}$ ), and $S\left(\lambda_{q}\right)$ as a function of $\lambda_{q}$. It must be admitted that from the present point of view this is by no means an obvious thing to do. We shall present what justifications we can, and attempt to state clearly the analyticity assumptions that we make.

In particular, the domains $\mathcal{D}_{0}, \mathcal{D}_{0}^{\prime}, \ldots, \mathcal{D}_{N-1}^{\prime}$ are made up of $N+1$ connected Riemann sheets of the cut $t_{q}$ plane of Figure 2. Together they are the beginnings of the full Riemann surface on which the functions live (and which we intend to discuss in a later paper). Here we only need these domains or sheets. In fact we only need the surface consisting of $\mathcal{D}_{0}$ and the adjacent neighbourhoods of $\mathcal{D}_{0}^{\prime}, \ldots, \mathcal{D}_{N-1}^{\prime}$ (obtained by just crossing the branch cuts in Figure 2). Since $\left|\lambda_{p}\right|<1$, and on $\mathcal{D}_{0}$ it is true that $\left|\lambda_{q}\right|<1$, we can choose this surface so that

$$
\left|\lambda_{p} \lambda_{q}\right|<1 \quad \text { on } \quad \mathcal{D}_{+}
$$

Let us call this extended surface $\mathcal{D}_{+}$. It includes the central domain $\mathcal{D}_{0}$ and all functions are defined on it by analytic continuation from $\mathcal{D}_{0}$. Then a basic assumption that we make is: 
ASSUMPTION 1: The function $T\left(x_{q}, y_{q}\right)$ is non-zero and analytic on $\mathcal{D}_{+}$.

This is consistent with the low-temperature result and is a standard assumption in the inversion relation method: the free energy is analytic in some fundamental domain that includes the physical regime. The remark after equation (7) that $\mu_{q}$ enters $T\left(x_{q}, y_{q}\right)$ only via its $N$ th power $\lambda_{q}$ is important here: it it were not true then there would also be branch cuts at 0 and $\infty$ in the complex $t_{q}$ plane, and the surface would be even more complicated.

In $[7,8]$ we took $L$ to be large but finite and used the fact that $\tau_{j}\left(t_{q}\right), S\left(\lambda_{q}\right)$ are then polynomials to solve the functional relations. Here we adopt a different (but related) strategy: we use Weiner-Hopf factorization to solve (22) - (28).

\section{Calculation of $\tau_{2}\left(t_{q}\right)$}

Since $\mathcal{D}_{+}$extends beyond the branch cuts in Fig. $2, \tau_{2}\left(t_{q}\right)$ is not necessarily a single-valued function of $t_{q}$ : we may expect it to have these branch cuts in the complex $t_{q}$-plane.

First consider possible poles or zeros. From Assumption 1 and (24), these can occur only when $\lambda_{q}=\lambda_{p}, x_{q}^{N}=x_{p}^{N}$ and $y_{q}^{N}=y_{p}^{N}$, or when $\lambda_{q}=1 / \lambda_{p}, x_{q}^{N}=y_{p}^{N}$ and $y_{q}^{N}=x_{p}^{N}$. The second possibility is excluded by (44), so we have to consider the first. Since $y_{p}, y_{q} \in \mathcal{R}_{0}$, this can only happen when $y_{q}=y_{q}$. But then the term $\left(t_{p}-t_{q}\right) /\left(x_{p}-x_{q}\right)$ simplifies to $y_{p}$ : the potential pole and zero cancel one another. Thus $\tau_{2}\left(t_{q}\right)$ is analytic and non-zero in $\mathcal{D}_{+}$. From $(22)$, so therefore is $\tau_{N}\left(t_{q}\right)$.

From (22) and (24),

$$
\tau_{N}\left(t_{q}\right)=\frac{\left(x_{p}-\omega^{-1} x_{q}\right)\left(y_{p}^{N}-x_{q}^{N}\right)\left(t_{p}^{N}-t_{q}^{N}\right) T\left(x_{q}, y_{q}\right)}{y_{p}^{2 N-2}\left(x_{p}^{N}-x_{q}^{N}\right)\left(y_{p}-x_{q}\right)\left(t_{p}-\omega^{-1} t_{q}\right) T\left(\omega^{-1} x_{q}, y_{q}\right)} .
$$

Also, from (10) and (33),

$$
\frac{1}{T\left(\omega^{-1} x_{q}, y_{q}\right)}=\frac{\left(x_{p}^{N}-x_{q}^{N}\right)\left(y_{p}^{N}-y_{q}^{N}\right)\left(t_{p}-\omega^{-1} t_{q}\right) T_{0}\left(y_{q}, x_{q}\right)}{N\left(x_{p}-\omega^{-1} x_{q}\right)\left(y_{p}-y_{q}\right)\left(t_{p}^{N}-t_{q}^{N}\right)} .
$$

Combining these together, there are several cancellations, leaving

$$
\tau_{N}\left(t_{q}\right)=\frac{\left(y_{p}^{N}-x_{q}^{N}\right)\left(y_{p}^{N}-y_{q}^{N}\right) T\left(x_{q}, y_{q}\right) T_{0}\left(y_{q}, x_{q}\right)}{N y_{p}^{2 N-2}\left(y_{p}-x_{q}\right)\left(y_{p}-y_{q}\right)} .
$$

Now $T_{0}\left(x_{q}, y_{q}\right)$ is the analytic continuation of $T\left(x_{q}, y_{q}\right)$ across the cut $M_{0}$, where $x_{q}$ and $y_{q}$ are both on the boundary of $\mathcal{R}_{0}$. Analytically continuing the rhs of (47) across this cut and then interchanging $x_{q}$ with $y_{q}$ leaves it unchanged, while also leaving $t_{q}$ unchanged. For a given $t_{q}$, these are the only possible values of $\left(x_{q}, y_{q}\right)$ in this neighbourhood. Thus in the neighbourhood of $M_{0}$, on either side of the cut, 
$\tau_{N}\left(t_{q}\right)$ is a single-valued function of $t_{q}$. There is therefore no need for the cut $M_{0}$ for this function: it can be removed.

From (22), the equation (23) can be written as

$$
\tau_{N}\left(t_{q}\right) \tau_{2}\left(\omega^{-1} t_{q}\right)=\alpha_{q} .
$$

The rhs of this equation is certainly is not a single-valued function of $t_{q}$ across the cut $M_{0}$ (when $\left.\lambda_{q} \rightarrow 1 / \lambda_{q}\right)$, so nor is $\tau_{2}\left(\omega^{-1} t_{q}\right)$. Hence the plane in which $\tau_{2}\left(t_{q}\right)$ is analytic must have $M_{N-1}$ as a branch cut.

ASSUMPTION 2: $\tau_{2}\left(t_{q}\right)$ is single-valued, with logarithmic derivative zero at infinity, in the $t_{q}$ plane containing only the cut $M_{N-1}$.

This is by no means obvious. It is consistent with $\tau_{N}\left(t_{q}\right)=$ $\tau_{2}\left(t_{q}\right) \cdots \tau_{2}\left(\omega^{N-2} t_{q}\right)$ not having the cut $M_{0}$, but is not implied by it. It is possible that $\tau_{2}\left(t_{q}\right)$ could have cuts other than $M_{N-1}$ that cancelled one another in the product function $\tau_{N}\left(t_{q}\right)$. On the other hand, it is the simplest assumption consistent with $\tau_{N}\left(t_{q}\right)$ not having $M_{0}$ as a branch cut, and again part of the spirit of the inversion relation method is to assume the simplest possible analytic structure. $^{3}$ Exhibiting the possible multi-valuedness of $\tau_{2}\left(t_{q}\right)$ by writing it as $\tau_{2}\left(x_{q}, y_{q}\right)$, our assumption implies that for $\left(x_{q}, y_{q}\right)$ on $\mathcal{D}_{+}$,

$$
\tau_{2}\left(x_{q}, y_{q}\right)=\tau_{2}\left(\omega^{r} y_{q}, \omega^{-r} x_{q}\right)
$$

for $r=0, \ldots, N-2$. So we are assuming these additional symmetries.

The requirement that the logarithmic derivative be zero at infinity follows from the finiteness and analyticity of the Boltzmann weights and hence $T\left(x_{q}, y_{q}\right)$ when $x_{q} \rightarrow \infty$ in $\mathcal{E}$.

It follows from these two assumptions and the above remarks that there is a closed curve $\mathcal{C}$ is the complex $t_{q}$ plane, surrounding the potential branch points $\eta, 1 / \eta$ as in Figure 2, such that

i) $\tau_{N}\left(t_{q}\right)$ is analytic and non-zero inside and on $\mathcal{C}$,

ii) $\tau_{2}\left(\omega^{-1} t_{q}\right)$ is analytic and non-zero outside and on $\mathcal{C}$,

iii) $\left(d / d t_{q}\right) \log \tau_{2}\left(\omega^{-1} t_{q}\right) \rightarrow 0$ as $t_{q} \rightarrow \infty$.

We can now solve (48) by Wiener-Hopf factorization. [25] Regard $\alpha_{q}$ as a function $\alpha\left(t_{q}\right)$ of $t_{q}$ and temporarily drop the suffix $q$. Define

$$
\begin{aligned}
& F_{-}(s)=-\frac{1}{2 \pi i} \oint_{\mathcal{C}} \frac{1}{t-s}\left(\frac{d}{d t} \log \alpha(t)\right) d t, \text { s outside } \mathcal{C}, \\
& F_{+}(s)=\frac{1}{2 \pi i} \oint_{\mathcal{C}} \frac{1}{t-s}\left(\frac{d}{d t} \log \alpha(t)\right) d t, \text { s inside } \mathcal{C} .
\end{aligned}
$$

Shifting the contour for $F_{-}(s)$ to be inside $\mathcal{C}$, that for $F_{+}(s)$ to be outside, and $s$ inbetween, it follows from Cauchy's integral formula that

$$
F_{-}(s)+F_{+}(s)=\alpha^{\prime}(s) / \alpha(s) .
$$

\footnotetext{
${ }^{3}$ We of course know that Assumption 2 is true from [7], but are trying to present an argument based on the inversion relation.
} 
Hence from (48),

$$
F_{-}(s)-\frac{d}{d s} \log \tau_{2}\left(\omega^{-1} s\right)=-F_{+}(s)+\frac{d}{d s} \log \tau_{N}(s) .
$$

The rhs of this equation is analytic for $s$ inside and on $\mathcal{C}$, while the lhs is analytic outside and on $\mathcal{C}$ and tends to zero as $s \rightarrow \infty$. Hence both sides are entire and bounded. By Liouville's theorem they must each be constant, in fact zero, so

$$
\frac{d}{d s} \log \tau_{2}\left(\omega^{-1} s\right)=F_{-}(s) .
$$

We can shrink $\mathcal{C}$ to just surround the branch cut in the $t$ plane from $\eta$ to $1 / \eta$. Then as $t$ goes round $\mathcal{C}, \lambda=\lambda_{q}$ (as defined by (29), goes anti-clockwise round the unit circle. Changing the variable of integration in (50) to $\theta$, where $\lambda=e^{i \theta}$, then replacing $s$ by $\omega t_{q}$, it follows that

$$
\log \tau_{2}\left(t_{q}\right)=\frac{1}{2 \pi} \int_{0}^{2 \pi}\left(\frac{1+\lambda_{p} e^{i \theta}}{1-\lambda_{p} e^{i \theta}}\right) \log \left[\frac{\Delta(\theta)-\omega t_{q}}{y_{p}^{2}}\right] d \theta
$$

for $t_{q}$ lying in the complex plane with a single branch cut from $\omega^{-1} \eta$ to $\omega^{-1} / \eta$. Here

$$
\Delta(\theta)=\left(\frac{1-2 k^{\prime} \cos \theta+k^{\prime 2}}{k^{2}}\right)^{1 / N}
$$

and we have integrated (53). We have fixed the integration constant by using (23) and the formula

$$
\frac{1}{2 \pi} \int_{0}^{2 \pi}\left(\frac{1+\lambda_{p} e^{i \theta}}{1-\lambda_{p} e^{i \theta}}\right) \log \left[\Delta(\theta)^{N}-t_{q}^{N}\right]=\log \left[\frac{k^{\prime}\left(1-\lambda_{p} \lambda_{q}\right)^{2}}{k^{2} \lambda_{q}}\right],
$$

which is true when $t_{q}, \lambda_{q}$ are related by (29) and $\left|\lambda_{p}\right|<1,\left|\lambda_{q}\right|<1$. It also follows from this formula and (48) that $\tau_{N}\left(t_{q}\right)=\tau_{2}\left(t_{q}\right) \cdots \tau_{2}\left(\omega^{N-2} t_{q}\right)$, in agreement with (22).

\section{Calculation of $S\left(\lambda_{q}\right)$}

The function $S\left(\lambda_{q}\right)$ is defined by (27) in the domain $\mathcal{D}_{0}$, where $\left|\lambda_{q}\right|$ is sufficiently small as to justify our neglect of various terms in the functional relations in the limit of $L$ large. (At low temperatures, this means that $\left|\lambda_{q}\right|<k^{\prime}$ ). Outside this domain we here define it by analytic continuation.

This is a different definition from [7], where much use was made of the fact that $S\left(\lambda_{q}\right)$ is a polynomial for finite $L$, with zeros located approximately on concentric circles between $\left|\lambda_{q}\right|=k^{\prime}$ and $\left|\lambda_{q}\right|=1 / k^{\prime}$. In the large- $L$ limit these cause the function $S\left(\lambda_{q}\right)$ of [7] to have a 
different analytic form across each such circle of zeros, so the two definitions will only agree for $\left|\lambda_{q}\right|$ inside the smallest circle.

We first repeatedly use $(24)$ to express $T\left(\omega x_{q}, y_{q}\right), \ldots, T\left(\omega^{N-1} x_{q}, y_{q}\right)$ in (27) in terms of $T\left(x_{q}, y_{q}\right)$, giving

$$
S\left(\lambda_{q}\right)=\xi_{q} T\left(x_{q}, y_{q}\right)^{N} \prod_{j=1}^{N-1}\left\{\frac{\left(y_{p}-\omega^{j} x_{q}\right)\left(t_{p}-\omega^{j-1} t_{q}\right)}{y_{p}^{2}\left(x_{p}-\omega^{j-1} x_{q}\right) \tau_{2}\left(\omega^{j-1} t_{q}\right)}\right\}^{N-j} .
$$

Within $\mathcal{D}_{0}$, if $\lambda_{q}=\mu_{q}^{N}$ is given, we take $y_{q}$ to be the root of (1) lying in $\mathcal{R}_{0}$. There are $N$ possible choices for $x_{q}$ in $\mathcal{E}$, but by the construction of $(27), S\left(\lambda_{q}\right)$ is the same for each, so it is a single-valued function of $\lambda_{q}$ in $\mathcal{D}_{0}$ and (by analytic continuation) in $\mathcal{D}_{+}$. From Assumption 1 and the definition (27), it is analytic and non-zero in $\mathcal{D}_{+}$.

For $\left|\lambda_{q}\right|$ close to one, choose $x_{q}$, like $y_{q}$, to lie near the boundary of $\mathcal{R}_{0}$. Replacing $\lambda_{q}$ by $1 / \lambda_{q}$ simply interchanges $x_{q}$ with $y_{q}$, while leaving $t_{q}$ unchanged. Note that $\tau_{2}\left(\omega^{-1} t_{q}\right)$ does not occur on the rhs of (57), so allowing $\lambda_{q}$ to move just outside the unit circle does not take any of the $\tau_{2}$ functions in (57) across a branch cut in the $t_{q}$ plane and they are unchanged by analytically continuing from $\lambda_{q}$ to $1 / \lambda_{q}$.

The function $T\left(x_{q}, y_{q}\right)$ is simply replaced by $T_{0}\left(y_{q}, x_{q}\right)$, which is given by (47). So analytically continuing (57) from $\lambda_{q}$ to $1 / \lambda_{q}$ (thereby interchanging $x_{q}$ with $y_{q}$ ), then multiplying by the original equation, we obtain (after many cancellations)

$$
S\left(\lambda_{q}\right) S\left(1 / \lambda_{q}\right)=N^{N}\left(\lambda_{p} / y_{p}^{2 N}\right)^{N-1} \prod_{j=1}^{N-1} \frac{\left(t_{p}-\omega^{j-1} t_{q}\right)^{2 N-2 j}}{\tau_{2}\left(\omega^{j-1} t_{q}\right)^{N-2 j}} .
$$

This is not the same as equation (26) of [7], because here $S\left(1 / \lambda_{q}\right)$ is defined by analytic continuation of $S\left(1 / \lambda_{q}\right)$ from $|\lambda|<1$ to $|\lambda|>1$. As remarked above, this is a different definition from that used in [7].

The rhs is known. As $\lambda_{q}$ moves round the unit circle, $t_{q}$ moves on the positive real axis from $\eta$ to $1 / \eta$ and back again. The logarithm of the rhs is analytic on this contour, returning to its original value. It follows from the above remarks that $\log S\left(\lambda_{q}\right)$ is analytic inside and on the unit circle in the complex $\lambda_{q}$-plane.

We can therefore solve (58) for $S\left(\lambda_{q}\right)$ by Wiener-Hopf factorization. Writing the rhs of (58) as $R\left[t_{q}\right]$, we obtain

$$
\log S\left(\lambda_{q}\right)=\frac{1}{4 \pi} \int_{0}^{2 \pi} \frac{1+\lambda_{q} e^{i \theta}}{1-\lambda_{q} e^{i \theta}} \log R[\Delta(\theta)],
$$

where $\Delta(\theta)$ is defined by (55). Define, ${ }^{4}$ for $\left|\lambda_{p}\right|<1$ and $\left|\lambda_{q}\right|<1$ :

$$
A_{p q}=\frac{1}{2 \pi} \int_{0}^{2 \pi} \frac{1+\lambda_{p} e^{i \theta}}{1-\lambda_{p} e^{i \theta}} \sum_{j=1}^{N-1}(N-j) \log \left\{\frac{k^{2 / N}\left[\Delta(\theta)-\omega^{j} t_{q}\right]}{1-\omega^{j}}\right\} d(\$ 0)
$$

${ }^{4}$ These definitions are the same as those of eqns. (45) and (46) of [7], except for the factor $k^{2 / N} /\left(1-\omega^{j}\right)$ inside the logarithm in $A_{p q}$. This gives an extra constant contribution $(N-1) \log k+i \pi(N-2)(N-1) / 12-(N / 2) \log N$ to $A_{p q}$, and ensures that $A_{p q}=0$ in the low-temperature limit of the Appendix. 


$$
\begin{aligned}
B_{p q}= & \frac{1}{8 \pi^{2}} \int_{0}^{2 \pi} \int_{0}^{2 \pi} \frac{1+\lambda_{p} e^{i \theta}}{1-\lambda_{p} e^{i \theta}} \frac{1+\lambda_{q} e^{i \phi}}{1-\lambda_{q} e^{i \phi}} \\
& \times \sum_{j=1}^{N-1}(N-2 j) \log \left[\omega^{-j / 2} \Delta(\theta)-\omega^{j / 2} \Delta(\phi)\right] d \theta d \phi,
\end{aligned}
$$

it follows that

$$
\log S\left(\lambda_{q}\right)=(N-1) \log \left[\frac{k^{\prime}\left(1-\lambda_{p} \lambda_{q}\right)^{2}}{k y_{p}^{N} \lambda_{p}^{1 / 2}}\right]-A_{q p}-B_{p q} .
$$

Let $\bar{q}=\left\{y_{q}, x_{q}, 1 / \lambda_{q}, t_{q}, 1 / \mu_{q}\right\}$, so $\lambda_{\bar{q}}=1 / \lambda_{q}$ and $t_{\bar{q}}=t_{q}$. One can verify that (62) does indeed satisfy (58) by analytically continuing $A_{q p}, B_{p q}$ to $\left|\lambda_{q}\right|>1$ (deforming the contours of integration accordingly), and verifying that the resulting functions satisfy

$$
\begin{gathered}
A_{q p}+A_{\bar{q} p}=2 \sum_{j=1}^{N-1}(N-j) \log \left[\frac{k^{2 / N}\left(t_{q}-\omega^{j} t_{p}\right)}{1-\omega^{j}}\right], \\
B_{p q}+B_{p, \bar{q}}=\sum_{j=1}^{N-1}(N-2 j) \log \left[\frac{\tau_{2}\left(\omega^{j-1} t_{q}\right)}{1-\omega^{j}}\right],
\end{gathered}
$$

provided that for $\lambda_{q}$ near (on) the unit circle, $t_{q}$ is taken to be the solution of (29) near (on) the real positive axis, so that $\left|\arg \left(t_{q}\right)\right|<$ $\pi / N$.

\section{Calculation of $T\left(x_{q}, y_{q}\right)$}

Now we know $S\left(\lambda_{q}\right)$, we can solve (57) for $\kappa_{p q}=T\left(x_{q}, y_{q}\right)$. The result is best expressed in terms of $T\left(x_{q}, y_{q}\right) /\left(\bar{D}_{p q} P_{p q}\right)^{1 / N}$, where $\bar{D}_{p q}, P_{p q}$ are defined in (35). From (2.44) of [19],

$$
\bar{D}_{p q}=\prod_{j=1}^{N-1}\left[\frac{\left(1-\omega^{j}\right)\left(t_{p}-\omega^{j} t_{q}\right)}{\left(x_{p}-\omega^{j} x_{q}\right)\left(y_{p}-\omega^{j} y_{q}\right)}\right]^{j},
$$

while from (3),

$$
P_{p q}=\left(\lambda_{p} / \lambda_{q}\right)^{(N-1) / 2} \prod_{j=1}^{N-1}\left[\frac{y_{q}-\omega^{j} x_{p}}{y_{p}-\omega^{j} x_{q}}\right]^{N-j} .
$$

Using these formulae and (28), (57) gives

$$
\begin{aligned}
\frac{T\left(x_{q}, y_{q}\right)^{N}}{\bar{D}_{p q} P_{p q}} & =\left(\lambda_{q}^{1 / 2} \alpha_{q}\right)^{1-N} S\left(\lambda_{q}\right) \prod_{j=1}^{N-1}\left[\frac{\tau_{2}\left(\omega^{j-1} t_{q}\right)}{1-\omega^{j}}\right]^{N-j} \\
& =S\left(\lambda_{q}\right) \exp \left(A_{p q}\right)\left[\frac{k y_{p}^{N} \lambda_{q}^{1 / 2}}{k^{\prime}\left(1-\lambda_{p} \lambda_{q}\right)^{2}}\right]^{N-1} \\
& =\left(\lambda_{q} / \lambda_{p}\right)^{(N-1) / 2} \exp \left(A_{p q}-A_{q p}-B_{p q}\right)
\end{aligned}
$$


This is the result (50) of [7].

There are two "inversion" relations: the inversion relation (37) and the rotation symmetry (39). We have derived (67) from the single combined relation (41). However, $B_{p q}=-B_{q p}$, so the rhs of (67) is inverted by interchanging $p$ with $q$. Thus (37) is indeed satisfied and we have in fact satisfied both relations.

\section{Analytic continuation of $T\left(x_{q}, y_{q}\right)$}

We can readily use the above equations to obtain the analytic continuation of $T\left(x_{q}, y_{q}\right)$ from $\mathcal{D}_{0}$ to the neighbouring domains $\mathcal{D}_{0}^{\prime}, \ldots, \mathcal{D}_{N-1}^{\prime}$. As in section 3 , if $\left(x_{q}, y_{q}\right) \in \mathcal{D}_{0}$ we write $T\left(x_{q}, y_{q}\right)$ simply as $T\left(x_{q}, y_{q}\right)$, while if $\left(x_{q}, y_{q}\right) \in \mathcal{D}_{r}^{\prime}$ (for $r=0, \ldots, N-1$ ) we write $T\left(x_{q}, y_{q}\right)$ as $T_{r}\left(x_{q}, y_{q}\right)$.

First note by repeated use of $(24)$ that, for $\left(x_{q}, y_{q}\right)$ in $\mathcal{D}_{0}$ and $r=0, \ldots, N-1$,

$$
T\left(\omega^{r} x_{q}, y_{q}\right)=T\left(x_{q}, y_{q}\right) \prod_{j=1}^{r} \frac{\left(y_{p}-\omega^{j} x_{q}\right)\left(t_{p}-\omega^{j-1} t_{q}\right)}{y_{p}^{2}\left(x_{p}-\omega^{j-1} x_{q}\right) \tau_{2}\left(\omega^{j-1} t_{q}\right)} .
$$

Now analytically continue $\left(x_{q}, y_{q}\right)$ from $\mathcal{D}_{0}$ to $\mathcal{D}_{0}^{\prime}$, so that $t_{q}$ goes through the branch cut $M_{0}$. Then $\left(\omega^{r} x_{q}, y_{q}\right)$ on the rhs goes from $\mathcal{D}_{0}$ through the cut $M_{r}$ to $\mathcal{D}_{r}^{\prime}$. The function $T\left(x_{q}, y_{q}\right)$ on the rhs therefore becomes the function $T_{0}\left(x_{q}, y_{q}\right)$, while $T\left(\omega^{r} x_{q}, y_{q}\right)$ on the rhs becomes $T_{r}\left(\omega^{r} x_{q}, y_{q}\right)$.

Going through the branch cut $M_{0}$ and returning to the original value of $t_{q}$ is equivalent to interchanging $x_{q}$ with $y_{q}$. (The branch points of $M_{0}$ are where $x_{q}=y_{q}$.) We can therefore interchange $x_{q}$ with $y_{q}$ and obtain the equation

$$
T_{r}\left(\omega^{r} y_{q}, x_{q}\right)=T_{0}\left(y_{q}, x_{q}\right) \prod_{j=1}^{r} \frac{\left(y_{p}-\omega^{j} y_{q}\right)\left(t_{p}-\omega^{j-1} t_{q}\right)}{y_{p}^{2}\left(x_{p}-\omega^{j-1} y_{q}\right) \tau_{2}\left(\omega^{j-1} t_{q}\right)} .
$$

Like (68), this equation holds for $\left(x_{q}, y_{q}\right)$ in $\mathcal{D}_{0}$ and $r=0, \ldots, N-$ 1. The functions $\tau_{2}\left(t_{q}\right), \ldots, \tau_{2}\left(\omega^{r-1} t_{q}\right)$ in these equations are singlevalued and analytic functions of $t_{q}$ across the cut $M_{0}$, i.e. $M_{0}$ is not a brach cut of these functions. Hence they are the same functions, with the same values, in each equation.

We can therefore eliminate the $\tau_{2}$ functions by dividing (69) by (68). Using also the form (33) of the inversion relation, we obtain, for $\left(x_{q}, y_{q}\right)$ in $\mathcal{D}_{0}$ and $r=0, \ldots, N-1$,

$$
\begin{aligned}
T_{r}\left(\omega^{r} y_{q}, x_{q}\right)= & \frac{N T\left(\omega^{r} x_{q}, y_{q}\right)}{T\left(\omega^{-1} x_{q}, y_{q}\right) T\left(x_{q}, y_{q}\right)} \prod_{j=1}^{r} \frac{t_{p}-\omega^{j-1} t_{q}}{\left(x_{p}-\omega^{j-1} y_{q}\right)\left(y_{p}-\omega^{j} x_{q}\right)} \\
& \times \prod_{j=r+1}^{N-1} \frac{t_{p}-\omega^{j-1} t_{q}}{\left(x_{p}-\omega^{j-1} x_{q}\right)\left(y_{p}-\omega^{j} y_{q}\right)}
\end{aligned}
$$


Given $T\left(x_{q}, y_{q}\right)$ in $\mathcal{D}_{0}$, this relation enables us to calulate the function in each neighbouring domain $\mathcal{D}_{r}^{\prime}$. If we set $r=0$ we regain the inversion relation (33), while if we set $r=N-1$ we obtain the alternative form (34) predicted in section 3.

One can continue. If we allow $x_{q}$ in (70) to move from $\mathcal{E}$ into $\mathcal{R}_{s}$ (with $s=1, \ldots, N-1)$, then $\left(x_{q}^{\prime}, y_{q}^{\prime}\right)=\left(\omega^{r} y_{q}, x_{q}\right)$ on the rhs moves into a new domain $\mathcal{D}_{r s}$, where $x_{q}^{\prime} \in \mathcal{E}$ and $y_{q}^{\prime} \in \mathcal{R}_{s}$. Correspondingly, $T_{r}\left(x_{q}^{\prime}, y_{q}^{\prime}\right)$ on the lhs becomes the function $T_{r s}\left(x_{q}^{\prime}, y_{q}^{\prime}\right)$. On the rhs, the $T$ functions become $T_{r+s}, T_{s-1}, T_{s}$, all of which can in turn be expressed in terms of the original function $T$ (in domain $\mathcal{D}_{0}$ ) by using (70).

The we can continue $x_{q}^{\prime}$ into $\mathcal{R}_{t}$ to obtain the function $T_{r s t}$ whose arguments lie in the domain $\mathcal{D}_{r s t}^{\prime}$, and so on. If each function were unique, then this procedure would give a Cayley tree of functions and domains, each having $N$ neighbours, with no circuits. This would form an infinite-dimensional Bethe lattice. In a subsequent paper we intend to show that in fact the functions are not unique and there are circuits, so that one obtains instead an $N$-dimensional lattice (for $N>2$ ), each site corresponding to a domain and a function. This is the Riemann surface on which $T\left(x_{q}, y_{q}\right)$ lives.

By contrast, the Boltzmann weights and related quantities of course form a zero-dimensional surface: for instance, the function $\bar{D}_{p q}$ in (65) has just $2 N$ sheets in the $t_{q}$ plane, corresponding to either $x_{q}$ or $y_{q}$ being in $\mathcal{R}_{r}$ (for $r=0, \ldots, N-1$ ), the other in $\mathcal{E}$.

We also hope to explore the question of whether one can describe the Riemann surface for $T\left(x_{q}, y_{q}\right)$ by an appropriate generalization of the hyperelliptic variables and functions defined in [10].

\section{Analytic continuation in $x_{p}, y_{p}$}

Up till now we have held the $p$ variables (corresponding to the vertical rapidity) fixed at some values in $\mathcal{D}_{0}$, except possibly in the inversion relations (36) - (38). One can of course vary the $p$ variables as well as the $q$ variables, and this would be necessary for a full understanding of the Riemann surface on which then free energy lives. In particular, we should like to obtain the $p$-variable analogue of (70).

From this point of view $T$ is a function $T\left(x_{p}, y_{p} \mid x_{q}, y_{q}\right)$ of both the $p$ and $q$ variables. It has an unexpected ratio property. From (54) it is apparent that $\tau_{2}\left(t_{q}\right)$ is unchanged by replacing $x_{p}, y_{p}$ by $\omega x_{p}, y_{p}$ (the variables remain in $\mathcal{D}_{0}$ and $\lambda_{p}$ is unaltered). From (24) it follows that, for $\left(x_{p}, y_{p}\right)$ and $\left(x_{q}, y_{q}\right)$ both in $\mathcal{D}_{0}$,

$$
\frac{T\left(x_{p}, y_{p} \mid x_{q}, y_{q}\right) T\left(\omega x_{p}, y_{p} \mid \omega x_{q}, y_{q}\right)}{T\left(x_{p}, y_{p} \mid \omega x_{q}, y_{q}\right) T\left(\omega x_{p}, y_{p} \mid x_{q}, y_{q}\right)}=\frac{\left(x_{p}-x_{q}\right)\left(\omega t_{p}-t_{q}\right)}{\left(\omega x_{p}-x_{q}\right)\left(t_{p}-t_{q}\right)}
$$

This result is not at all obvious from first principles: it could serve as a useful test of series expansions. 
Note that one has to be very careful in applying the equations of this paper in domains other than those for which they were obtained. This relation is a good example: if we analytically continue $T\left(x_{p}, y_{p} \mid x_{q}, y_{q}\right)$ to $\left(x_{p}, y_{p}\right) \in \mathcal{D}_{0},\left(x_{q}, y_{q}\right) \in \mathcal{D}_{r}^{\prime}$ by allowing $x_{q}$ to move from $\mathcal{E}$ to $\mathcal{R}_{r}, y_{q}$ from $\mathcal{R}_{0}$ to $\mathcal{E}$, then the $T$-functions with third argument $x_{q}$ will become the functions $T_{r}$ above, while those with third argument $\omega x_{q}$ will become $T_{r+1}$. The different functions, originally on the same sheet, will move onto different sheets.

For the rest of this section we shall hold $x_{q}, y_{q}$ fixed, lying in $\mathcal{D}_{0}$, so for brevity we write $T\left(x_{p}, y_{p} ; x_{q}, y_{q}\right)$ as $T\left[x_{p}, y_{p}\right]$.

Fortunately it is not necessary to repeat all the above working. We can replace $x_{p}, y_{p}$ in the final result (67) by $\omega x_{p}, y_{p}$ (so again the variables $x_{p}, y_{p}$ remain in $\mathcal{D}_{0}$ ) and take the ratio of the equation with these arguments to that with the original arguments. Since $\lambda_{p}$ is the same in both, the factors involving $A_{p q}, B_{p q}$ cancel, and we obtain

$$
\hat{\tau}_{2}\left(t_{p}\right)=\frac{\left(t_{q}-\omega t_{p}\right)\left(y_{q}-\omega x_{p}\right)}{y_{q}^{2}\left(x_{q}-\omega x_{p}\right)} \frac{T\left[\omega x_{p}, y_{p}\right]}{T\left[x_{p}, y_{p}\right]}
$$

where $\hat{\tau}_{2}\left(t_{p}\right)$ is the function $\tau_{2}\left(t_{q}\right)$ defined by $(54)$, but with $p$ interchanged with $q$, i.e.

$$
\log \hat{\tau}_{2}\left(t_{p}\right)=\frac{1}{2 \pi} \int_{0}^{2 \pi}\left(\frac{1+\lambda_{q} e^{i \theta}}{1-\lambda_{q} e^{i \theta}}\right) \log \left[\frac{\Delta(\theta)-\omega t_{p}}{y_{q}^{2}}\right] d \theta .
$$

It is analytic in the $t_{p}$ plane, except for a single branch cut from from $\omega^{-1} \eta$ to $\omega^{-1} \eta^{-1}$. This result is of course consistent with (71).

Let $T_{r}\left[x_{p}, y_{p}\right]$ be the analytic continuation of $T\left[x_{p}, y_{p}\right]$ from $\left(x_{p}, y_{p}\right) \in$ $\mathcal{D}_{0}$ to $\left(x_{p}, y_{p}\right) \in \mathcal{D}_{r}^{\prime}$ (i.e. $x_{p}$ moves to the region $\mathcal{R}_{r}$ of Figure $3, y_{p}$ to the region $\mathcal{E})$. Then from the inversion relations $(36)$ - (38),

$$
T\left[\omega^{-1} y_{p}, x_{p}\right] T_{0}\left[x_{p}, y_{p}\right]=r_{p q}=T_{N-1}\left[\omega^{-1} y_{p}, x_{p}\right] T\left[x_{p}, y_{p}\right] .
$$

(The second of these relations is the analytic contination of the first.)

Proceeding as in the previous section, from (72) and either equation of (74), we can deduce that

$$
\begin{aligned}
T_{r}\left[\omega^{r} y_{p}, x_{p}\right]= & \frac{N T\left[\omega^{r} x_{p}, y_{p}\right]}{T\left[\omega^{-1} x_{p}, y_{p}\right] T\left[x_{p}, y_{p}\right]} \prod_{j=1}^{r} \frac{t_{q}-\omega^{j} t_{p}}{\left(x_{q}-\omega^{j} x_{p}\right)\left(y_{q}-\omega^{j} y_{p}\right)} \\
& \times \prod_{j=r+1}^{N-1} \frac{t_{q}-\omega^{j} t_{p}}{\left(x_{q}-\omega^{j} y_{p}\right)\left(y_{q}-\omega^{j} x_{p}\right)}
\end{aligned}
$$

for $r=0, \ldots, N-1$.

If $r=0$ we regain the first inversion relation (74); if $r=N-1$ we obtain the second. 


\section{Summary}

A basic difficulty with the $N$-state chiral Potts model is that it lacks the "rapidity-difference" property [8]. Because of this, there is no known explicit parametrization in terms of elliptic or other singlevalued single-variable functions. The author did show that one could parametrize the Boltzmann weights in terms of hyperelliptic functions with $N-1$ related arguments [10], but so far little progress has been made using this parametrization. (For $N=3$ it has been used to obtain the $f_{p q}$. [16]) For this reason, it is difficult to see how to use the standard, usually very simple, "inversion relation" method [4, 23] to obtain the free energy.

Here we have addressed this problem. We have shown that the inversion and rotation relations (37) and (39) give (41), and that this is sufficient to deduce the "infinite lattice functional relations" (22) (28), which from this point of view are simply definitions and direct consequences of the definitions.

We then made two assumptions as to the analyticity properties of the functions, and showed that these were sufficient to evaluate the free energy of the model by Wiener-Hopf factorization. The first assumption is very plausible and perfectly typical of the inversion relation method, which needs such assumptions to complete it. The second is by no means so obvious, implying as it does the symmetry (49). We have presented an argument for it - namely that $M_{0}$ is not a branch cut in the $t_{q}$ plane for the function $\tau_{N}\left(t_{q}\right)=\tau_{2}\left(t_{q}\right) \cdots \tau_{2}\left(\omega^{N-2} t q\right)$, and the easiest way to ensure this is to require that $\tau_{2}\left(t_{q}\right)$ only have the cut $M_{N-1}$. However, we must admit it is unlikely that we should have made such an assumption if we had not already known that it was implied by the exact finite-size functional relation method used in [7] and $[8]$.

In Appendix B we present for reference the solution for the $N=2$ Ising case, where the standard elliptic function methods work immediately. However, in this case Assumption 2 is redundant, being a direct consequence of $\tau_{N}\left(t_{q}\right)$ not having $M_{0}$ as a branch cut.

In later papers we intend to obtain the full Riemann surface, and all poles and zeros, of $T\left(x_{p}, y_{p} \mid x_{q}, y_{q}\right)$ from (70), (71) and (75). This gives a very full description of the function and should greatly extend our understanding of it.

\section{Appendix A: checking the equations}

When performing the intricate calculations necessary to derive equations such as (54), (62), one encounters many irritating and uninteresting factors that are independent of $q$. Two checks on these can be obtained from the invariances discussed near the end of section 2 : these can be used to immediately check that the powers of $k$ and $y_{p}$ 
are correct. In particular, $\tau_{2}\left(t_{q}\right)$ is proportional to $y_{p}^{-2}$, while $\xi_{q}, S\left(\lambda_{q}\right)$ are proportional to $y_{p}^{-N(N-1)}$. The functions $T\left(x_{q}, y_{q}\right)$ and $T\left(y_{q}, x_{q}\right)$ do not contain any such external $y_{p}$ factors.

We are still left with constant factors coming from products of powers of $\pm \omega^{j}$ and $1-\omega^{j}$. To check these, the author has found it helpful to consider the low-temperature limit in which $k^{\prime} \rightarrow 0, \lambda_{p}$ and $\lambda_{q}$ are finite, and $x_{p}, y_{p}, x_{q}, y_{q} \rightarrow k^{-1 / N}, t_{p}, t_{q} \rightarrow k^{-2 / N}$. One needs to first eliminate zero differences like $y_{q}-x_{p}, t_{p}-t_{q}$ by using exact formulae such as

$$
y_{q}^{N}-x_{p}^{N}=\frac{k^{\prime}\left(1-\lambda_{p} \lambda_{q}\right)}{k \lambda_{p}}, t_{p}^{N}-t_{q}^{N}=\frac{k^{\prime}\left(\lambda_{p}-\lambda_{q}\right)\left(1-\lambda_{p} \lambda_{q}\right)}{k^{2} \lambda_{p} \lambda_{q}} .
$$

One can also use formulae such as

$$
\prod_{j=1}^{N-1}\left(1-\omega^{j}\right)=N, \prod_{j=1}^{N-1}\left(1-\omega^{j}\right)^{j}=N^{N / 2} e^{i \pi(N-2)(N-1) / 12}
$$

(though we have tried to arrange the expressions so that only the first of these is needed).

In this limit $T\left(x_{q}, y_{q}\right)=T\left(y_{q}, x_{q}\right)=1$ and

$$
\tau_{2}\left(\omega^{j-1} t_{q}\right)=\left(1-\omega^{j}\right) /\left(k^{2 / N} y_{p}^{2}\right) \text { for } j=1, \ldots, N-1 \text {, }
$$

while from (23) and (9),

$$
\tau_{2}\left(\omega^{-1} t_{q}\right)=\frac{k^{\prime}\left(1-\lambda_{p} \lambda_{q}\right)^{2}}{N k^{2 / N} y_{p}^{2} \lambda_{q}} .
$$

It follows from (28) that

$$
\xi_{q}=\left\{\frac{k^{\prime}\left(1-\lambda_{p} \lambda_{q}\right)}{k y_{p}^{N} \lambda_{p}^{1 / 2}}\right\}^{N-1}
$$

and from (57) that

$$
S\left(\lambda_{q}\right)=\xi_{q}\left(1-\lambda_{p} \lambda_{q}\right)^{N-1}=\left\{\frac{k^{\prime}\left(1-\lambda_{p} \lambda_{q}\right)^{2}}{k y_{p}^{N} \lambda_{p}^{1 / 2}}\right\}^{N-1}
$$

The rhs of (58) is now

$$
\left\{\frac{k^{\prime}\left(\lambda_{p}-\lambda_{q}\right)\left(1-\lambda_{p} \lambda_{q}\right)}{k y_{p}^{N} \lambda_{p}^{1 / 2} \lambda_{q}}\right\}^{2 N-2}
$$

and we see that (58) is indeed satisfied in this low-temperature limit.

Also, from (60),

$$
A_{p q}=B_{p q}=0
$$

so (A5) agrees with (62).

From (65) and (66),

$$
\bar{D}_{p q}=1, P_{p q}=\left(\lambda_{p} / \lambda_{q}\right)^{(N-1) / 2},
$$

so (67) is satisfied. 


\section{Appendix B: The Ising case}

When $N=2$, as in [15], the parameters $a_{q}, b_{q}, c_{q}, d_{q}$ of $([18])$ can be written as

$$
a_{q}, b_{q}, c_{q}, d_{q}=-H\left(u_{q}\right),-H_{1}\left(u_{q}\right), \Theta_{1}\left(u_{q}\right), \Theta\left(u_{q}\right),
$$

from which it follows that $x_{q}, y_{q}, \mu_{q}$ are

$$
x_{q}=-\sqrt{k} \operatorname{sn} u_{q}, y_{q}=-\sqrt{k} \frac{\operatorname{cn} u_{q}}{\operatorname{dn} u_{q}}, \mu_{q}=\frac{\sqrt{k^{\prime}}}{\operatorname{dn} u_{q}} .
$$

Here $H(u), H_{1}(u), \Theta(u), \Theta_{1}(u), \operatorname{sn} u_{q}, \operatorname{cn} u_{q}, \operatorname{dn} u_{q}$ are the usual Jacobi elliptic functions of modulus $k$. For $\left(x_{p}, y_{p}\right),\left(x_{q},\left(y_{q}\right) \in \mathcal{D}_{0}\right.$ we can restrict $u_{p}$ and $u_{q}$ to lie in the corresponding rectangle in Figure 4, i.e. $3 K / 2<\Re\left(u_{q}\right)<5 K / 2,-K^{\prime}<\Im\left(u_{q}\right) \leq K^{\prime}$. (The variables and functions $x_{q}, y_{q}, t_{q}, \lambda_{q}, T\left(x_{q}, y_{q}\right), \tau_{2}\left(t_{q}\right), S\left(\lambda_{q}\right)$ in this Appendix are all periodic of period $2 i K^{\prime}$.) Similarly, $\left(x_{q}, y_{q}\right) \in \mathcal{D}_{0}^{\prime}$ (or $\mathcal{D}_{1}^{\prime}$ ) if $u_{q}$ lies in the rectangle $\mathcal{D}_{0}^{\prime}$ (or $\mathcal{D}_{1}^{\prime}$ ) in Figure 4 . The vertical lines in between the rectangles are where $\left|\lambda_{q}\right|=1$.

Defining

$$
\operatorname{scd} u=\frac{\operatorname{sn} u}{\operatorname{cn} u \operatorname{dn} u}, h_{1}(u)=H_{1}(u / 2) \Theta_{1}(u / 2),
$$

it follows that

$$
W_{p q}(1)=k^{\prime} \operatorname{scd}\left(\frac{K-u}{2}\right), \bar{W}_{p q}(1)=k^{\prime} \operatorname{scd}\left(\frac{u}{2}\right),
$$

where $K\left(K^{\prime}\right)$ is the complete elliptic integral of modulus $k\left(k^{\prime}\right)$ and

$$
u=u_{q}-u_{p}
$$

and by definition $W_{p q}(0)=\bar{W}_{p q}(0)=1$.

We note that the Boltzmann weights depend on $u_{p}, u_{q}$ only via their difference $u_{q}-u_{p}$. This is the "difference property" for this model (it only holds for $N=2$ ). The partition function, transfer matrix and hence $T\left(x_{q}, y_{q}\right)$ therefore also depend on $p$ and $q$ only via $u=u_{q}-u_{p} ;$ in particular we can write $T\left(x_{q}, y_{q}\right)$ as $T(u)$.

From (10),

$$
r_{p q}=r(u)=\frac{h_{1}(0) \Theta_{1}(0) H_{1}(u)}{h_{1}(u)^{2}} .
$$

Incrementing $u_{q}$ by $K$ takes $x_{q}, y_{q}$ to $y_{q},-x_{q}$. Hence the inversion relation (25) becomes

$$
T(u) T(u+K)=r(u) .
$$

Define

$$
z=e^{-\pi u / K^{\prime}}, \quad q^{\prime}=e^{-\pi K / K^{\prime}}
$$




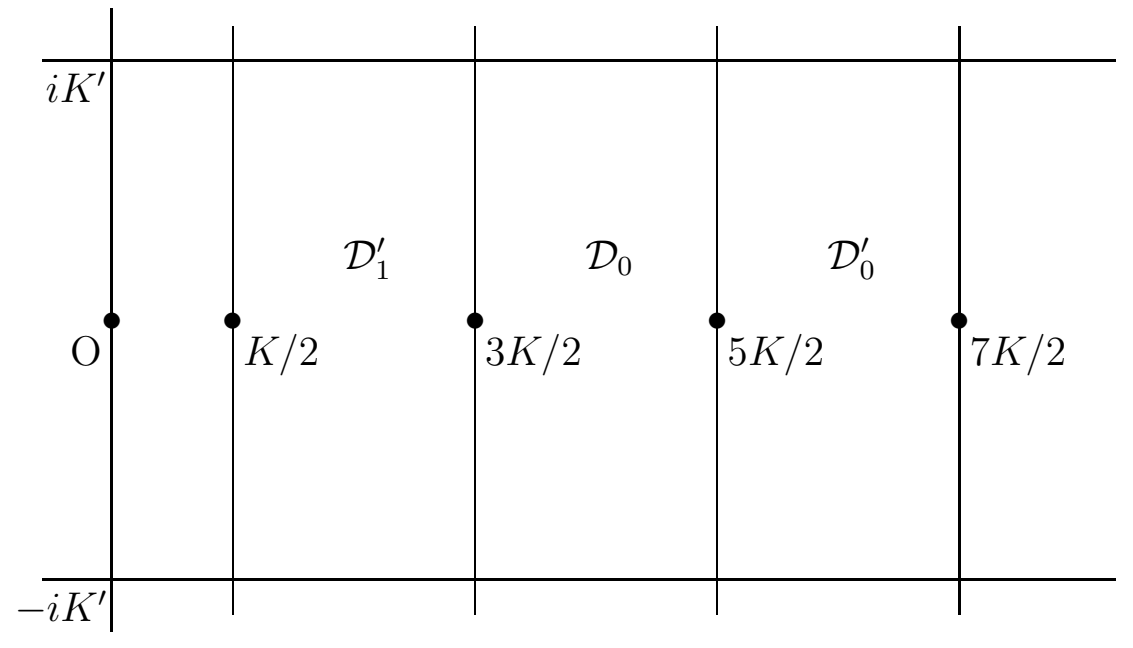

Figure 4: The complex $u_{q}$ plane in the Ising case, showing the rectangles corresponding to $\mathcal{D}_{0}, \mathcal{D}_{0}^{\prime}, \mathcal{D}_{1}^{\prime}$.

Our assumption 1 implies that $\log T(u)$ is analytic in the vertical strip $0 \leq \Re(u) \leq K$ in the complex $u$-plane, and periodic of period $2 i K^{\prime}$. Hence in this strip it has a Fourier expansion of the form

$$
\log T(u)=\sum_{m=-\infty}^{\infty} \rho_{m} z^{m}
$$

Taking logs of (B6), using this expansion and equating Fourier coefficients, we obtain

$$
\log T(u)=\sum_{m=1}^{\infty} \frac{\left(q^{\prime m}-q^{2 m}\right)\left(1-z^{m}\right)\left(1-q^{\prime m} / z^{m}\right)}{m\left(1+q^{\prime m}\right)^{2}\left(1+q^{\prime 2 m}\right)},
$$

which can be shown to be Onsager's result [1] for the free energy of the Ising model. If we define

$$
g(z)=\prod_{m=0}^{\infty} \frac{\left(1-q^{4 m+1} z\right)^{4 m+1}\left(1-q^{\prime 4 m+3} z\right)^{4 m+4}}{\left(1-q^{\prime 4 m+2} z\right)^{4 m+3}\left(1-q^{\prime 4 m+4} z\right)^{4 m+4}}
$$

then we can write (B9) as

$$
T(u)=g(z) g\left(q^{\prime} / z\right) /\left[g(1) g\left(q^{\prime}\right)\right],
$$

which manifests the rotation symmetry (39), i.e. $T(u)=T(K-u)$.

The auxiliary functions $\tau_{2}\left(t_{q}\right), S\left(\lambda_{q}\right)$ simplify if we define

$$
\tilde{T}(u)=h_{1}(u) h_{1}(K-u) T(u) /\left[h_{1}(0) h_{1}(K)\right],
$$

then

$$
\tau_{2}\left(t_{q}\right)=\frac{2 h_{1}(K)^{2} \tilde{T}\left(u_{q}-u_{p}\right) \tilde{T}\left(5 K-u_{q}-u_{p}\right)}{H_{1}\left(u_{p}\right)^{2} \Theta\left(u_{q}\right) \Theta_{1}\left(u_{q}\right)}
$$




$$
S\left(\lambda_{q}\right)=\frac{4 h_{1}(K)^{2} \tilde{T}\left(u_{q}-u_{p}\right) \tilde{T}\left(4 K-u_{q}-u_{p}\right)}{\left[H_{1}\left(u_{p}\right) \Theta\left(u_{q}\right)\right]^{2}} .
$$

The first of these formulae explicitly manifests the invariance of $\tau_{2}\left(t_{q}\right)$ under $u_{q} \rightarrow 5 K-u_{q}$, corresponding to interchanging $\mathcal{D}_{0}$ with $\mathcal{D}_{0}^{\prime}$ and $x_{q}$ with $y_{q}$. The second manifests the invariance of $S\left(\lambda_{q}\right)$ under $u_{q} \rightarrow 4 K-u_{q}$, corresponding to negating $x_{q}, t_{q}$ in $\mathcal{D}_{0}$, while leaving $\mu_{q}, \lambda_{q}, y_{q}$ unchanged. Note that

$$
h_{1}(K)^{2}=H_{1}(0)^{2} \Theta(0) \Theta_{1}(0) / 2 .
$$

There are further simplifications from using $\tilde{T}(u)$ instead of $T(u)$. The original inversion and rotation relations (37), (39) become

$$
\tilde{T}(u) \tilde{T}(-u)=\frac{H_{1}(u)^{2}}{H_{1}(0)^{2}}, \quad \tilde{T}(u)=\tilde{T}(K-u),
$$

while (B11) becomes

$$
\tilde{T}(u)=e^{\pi u(K-u) / 4 K K^{\prime}} \tilde{g}(z) \tilde{g}\left(q^{\prime} / z\right) /\left[\tilde{g}(1) \tilde{g}\left(q^{\prime}\right)\right],
$$

where $\tilde{g}(z)$ is a considerably simpler function than $g(z)$, being

$$
\tilde{g}(z)=\prod_{n=1}^{\infty}\left(\frac{1-q^{2 n-1} z}{1-q^{\prime 2 n} z}\right)^{2 n}
$$

It is also true that

$$
\tilde{T}(u)=\frac{H_{1}(u)}{H_{1}(0)} e^{\pi u / 4 K^{\prime}} \prod_{n=1}^{\infty} \frac{\left(1-q^{2 n-1} z\right)^{2 n-1}\left(1-q^{\prime 2 n} z^{-1}\right)^{2 n}}{\left(1-q^{\prime 2 n-1} z^{-1}\right)^{2 n-1}\left(1-q^{\prime 2 n} z\right)^{2 n}} .
$$

\section{References}

[1] L. Onsager, Crystal statistics. I. A two-dimensional model with an order-disorder transition, Phys. Rev 65 (1944) 117 149 .

[2] B. Kaufman, Crystal statistics. II. Partition function evaluated by spinor analysis, Phys. Rev. 76 (1949) 1232 - 1243.

[3] P.W. Kasteleyn, Dimer statistics and phase transitions, J. Math. Phys. 4 (1963) $287-293$.

[4] Yu.G. Stroganov, A new calculation method for partition functions in some lattice models, Phys. Lett. A 74 (1979) 116 -118 .

[5] R.J. Baxter and I.G. Enting, 399th solution of the Ising model, J. Phys. A 11 (1978) 2463 -2473.

[6] R.J. Baxter, Free energy of the solvable chiral Potts model, J. Stat. Phys. 52 (1988) $639-667$. 
[7] R.J. Baxter, Calculation of the eigenvalues of of the transfer matrix of the chiral Potts model, Proc. Fourth Asia-Pacific Physics Conference (Seoul, Korea, 1990), World-Scientific, Singapore (1), 1991, 42 - 58.

[8] R.J. Baxter, Chiral Potts model: eigenvalues of the tranfer matrix, Phys. Lett. A 146 (1990) 110 - 114.

[9] R.J. Baxter, Equivalence of the two results for the free energy of the chiral Potts model, J. Stat. Phys. 98 (2000) $513-535$.

[10] R.J. Baxter, Hyperelliptic function parametrization for the chiral Potts model, Proc. Int. Congress Mathematicians (Kyoto, Japan, 1990), Springer-Verlag, Tokyo, 1991, 1305 - 1317.

[11] R.J. Baxter, Rogers-Ramanujan identities in the hard hexagon model, J. Stat. Phys. 26 (1981) $427-452$.

[12] S. Howes, L.P. Kadanoff and M. den Nijs, Quantum model for commensurate-incommensurate transitions, Nucl. Phys. B 215[FS7] (1983) $169-208$.

[13] G. Albertini, B.M. McCoy, J.H.H. Perk and S. Tang, Excitation spectrum and order parameter for the integrable $N$-state chiral Potts model, Nucl. Phys. B 314 (1989) 741 - 763.

[14] M. Henkel and J. Lacki, Integrable chiral $Z_{n}$ quantum chains and a new class of trigonometric sums, Phys. Lett. A 138 (1989) $105-109$.

[15] R.J. Baxter, Functional relations for the order parameters of the chiral Potts model, J. Stat. Phys. 91 (1998) 499 - 524.

[16] R.J. Baxter, Some hyperelliptic function identities that occur in the chiral Potts model, J. Phys. A 31 (1998) $6807-6818$.

[17] R.J. Baxter, Functional relations for the order parameters of the chiral Potts model: low temperature expansions, Physica A 260 (1998) $117-130$.

[18] R.J. Baxter, J.H.H. Perk and H. Au-Yang, New solutions of the star-triangle relations for the chiral Potts model, Phys. Lett. A 128 (1988) 138 - 142.

[19] R.J. Baxter, V.V. Bazhanov and J.H.H. Perk, Functional relations for transfer matrices of the chiral Potts model, Int. J. Mod. Phys. B 4 (1990) $803-870$.

[20] V.V. Bazhanov and Yu.G. Stroganov, Chiral Potts model as a descendant of the six-vertex model, J. Stat. Phys. 59 (1990) $799-817$.

[21] R.J. Baxter, Chiral Potts model with skewed boundary conditions, J. Stat. Phys. 73 (1993) $461-495$.

[22] R.J. Baxter, Exactly Solved Models, in Fundamental Problems in Statistical Mechanics V (ed. E.G.D. Cohen), NorthHolland, Amsterdam, 1980, 109 - 141. 
[23] R.J. Baxter, The inversion relation method for some twodimensional exactly solved models in lattice statistics, J. Stat. Phys. 28 (1982) $1-41$.

[24] R.J. Baxter, Exactly Solved Models in Statistical Mechanics, Academic, London, 1982.

[25] B. Noble, Methods Based on the Wiener-Hopf Technique, Pergamon, London, 1958. 